\title{
INVERSE LIMITS OF TOPOLOGICAL GROUP COHOMOLOGIES
}

\author{
ARNOLD J. INSEL
}

\begin{abstract}
For second countable locally compact almost connected groups $G$ and $A$, where $A$ is abelian and $G$ acts on $A$ continuously, it is shown that it is possible to represent $A$ as an inverse limit of Lie groups $\left\{A_{n}\right\}$ compatible with the action of $G$ and such that $H^{*}(G, A)$ is isomorphic to $\lim _{n}$ inv $H^{*}\left(G, A_{n}\right)$, provided that $A$ is compact or connected.
\end{abstract}

1. Introduction. Let $G$ and $A$ be locally compact second countable groups with $A$ abelian. Suppose $G$ acts on $A$ continuously. In [4] a group cohomology is developed with this situation in mind, $H^{*}(G, A)$. We assume the definitions and results of [4].

To begin our study we approximate $A$ by an inverse sequence $\left\{A_{n}\right\}$ of Lie groups such that $G$ acts on each $A_{n}$ in a manner "compatible" with the inverse sequence. We next observe that the inverse sequence $\left\{A_{n}\right\}$ induces an inverse sequence $\left\{H^{*}\left(G, A_{n}\right)\right\}$ of cohomology groups. Considering the "natural" homomorphism of $H^{*}(G, A)$ to $\lim _{n} \operatorname{inv}\left\{H^{*}\left(G, A_{n}\right)\right\}$ we investigate the conditions under which the homomorphism is an isomorphism. We find sufficient conditions when $A$ is compact or connected.

2. Results. Throughout this paper $A$ and $G$ are locally compact, second countable, Hausdorff, almost connected topological groups and we assume a fixed action of $G$ on $A$. A group $G$ is almost connected provided that $G / G_{0}$ is compact, where $G_{0}$ is the connected component of $G$.

THEOREM 1. For any neighborhood $U$ of the identity of $A$ there exists a compact subgroup $K$ of $A$ such that

(1) $K \subset U$,

(2) $K$ is $G$-invariant,

(3) $A / K$ is a Lie group.

Proof. Let $E=A$ (S) $G$, be the semidirect product of $A$ and $G$ with respect to the action of $G$ on $A$. Note that topologically $E$ is the product of $A$ and $G$. We first observe that $E / E_{0}$ is almost connected. Let $A_{0}, G_{0}$ and $E_{0}$ be the components of $A, G$ and $E$ respectively. It is easily seen that $E_{0}=A_{0} \times G_{0}$. Also $E / A_{0} \times G_{0}$ is topologically homeomorphic to $A / A_{0} \times G / G_{0}-$ a compact space. Thus $E / E_{0}$ is compact. Thus $E$ is almost connected. Let $U$ be any neighborhood of the identity of $A$. Then $U \times G$ is a neighborhood of the

Presented to the Society, January 17, 1974 under the title On inverse limits in cohomology groups of topological groups; received by the editors January 8, 1975.

AMS (MOS) subject classifications (1970). Primary 22D99, 18H10; Secondary 22E99.

Key words and phrases. Inverse limit of Lie groups, topological group cohomologies. 
identity on $E$. Choose a smaller neighborhood $V \times W$ such that $V \times W$ $\subset U \times G$ and $V \times W$ has compact closure in $E$. Since $E$ is almost connected we conclude by [3] that there exists a closed normal subgroup $N$ of $E$ such that $N \subset V \times W$ and $E / N$ is a Lie group. Let $K^{\prime}=A \times\{1\} \cap N$ where 1 is the identity of $G$. Let $K$ be the projection of $K^{\prime}$ into $A$. Clearly $K \subset U, K$ is compact and $G$-invariant. By [2, p. 144] we obtain

$$
A / K \simeq \frac{A \times\{1\}}{K \times\{1\}}=\frac{A \times\{1\}}{(A \times\{1\}) \cap N} \simeq \frac{(A \times\{1\}) \cdot N}{N} .
$$

Since the right-most group is a closed subgroup of the Lie group $E / N, A / K$ is a Lie group.

COROLlaRY 1. There exists a decreasing sequence $\left\{U_{n}\right\}$ of neighborhoods of the identity of $A$ and a decreasing sequence of compact $G$-invariant subgroups $\left\{K_{n}\right\}$ of $A$ such that

(1) $\left\{U_{n}\right\}$ is a decreasing base for the neighborhood system of the identity,

(2) $K_{n} \subset U_{n}$ for all $n$,

(3) $A / K_{n}$ is a Lie group for all $n$.

Proof. The above corollary follows immediately from Theorem 1 . The only difficulty is to make $\left\{K_{n}\right\}$ into a decreasing sequence. But this is immediate once we observe by [3] that if $A / J$ and $A / K$ are Lie groups so is $A / J \cap K$. Use Theorem 1 to obtain a sequence $J_{n}$ satisfying (2) and (3) (but not necessarily decreasing). Then define $K_{n}=\bigcap_{i=1}^{n} J_{i}$ for all $n$.

Corollary 2. There exists a sequence of abelian Lie groups $\left\{A_{n}\right\}$ and continuous surjective homomorphisms $\left\{\pi_{n}\right\}$ and $\left\{\rho_{n}\right\}$ such that:

(1) $G$ acts on $A_{n}$ for all $n$.

(2) $\pi_{n}: A_{n} \rightarrow A_{n-1}$ and $\pi_{n}(\mathrm{~g} a)=g \pi_{n}(a)$ for all $a \in A_{n}$ and $g \in G$.

(3) $\rho_{n}: A \rightarrow A_{n}$ and $\rho_{n}(g a)=g \rho_{n}(a)$ for all $a \in A_{n}$ and $g \in G$.

(4) $\pi_{n} \rho_{n}=\pi_{n-1}$ for all $n$.

(5) Define $\phi: A \rightarrow \lim _{n}$ inv $A_{n}$ by $\phi(a)=\left\{\rho_{n}(a)\right\}$. Then $\phi$ is a topological isomorphism and $\phi(\mathrm{ga})=\mathrm{g} \phi(a)$ for all $a \in A$ and $g \in G$.

Proof. Referring to Corollary 1 define $A_{n}=A / K_{n}$ for all $n$. For each $n$ let $\rho_{n}: A \rightarrow A_{n}$ be the natural quotient map and for each $n$ let $\pi_{n}: A_{n} \rightarrow A_{n-1}$ be the map $\pi_{n}\left(x \cdot K_{n}\right)=x \cdot K_{n-1}, x \in A$. Clearly the corollary follows.

For the rest of this paper we assume the inverse system of Lie groups as constructed in Corollary 2.

On the cohomology level we inherit an inverse sequence $\left\{H^{*}\left(G A_{n}\right)\right\}$ with homomorphisms $\left\{\pi_{n_{*}}\right\}$ and $\left\{\rho_{n_{*}}\right\}$ induced by the homomorphisms $\left\{\pi_{n}\right\}$ and $\left\{\rho_{n}\right\}$ such that $\pi_{n_{*}} \rho_{n_{*}}=\pi_{n-1_{*}}$ for all $n$. For each $k$ define

$$
\Phi_{A}: H^{k}(G, A) \rightarrow \lim _{n} \operatorname{inv}\left\{H^{k}\left(G, A_{n}\right)\right\}
$$

by

$$
\Phi_{A}(h)=\left\{\rho_{n_{*}}(h)\right\}, \quad h \in H^{k}(G, A) .
$$

The next theorem will enable us to conclude that $\Phi_{A}$ is onto. To facilitate its proof let us recall some notation. For each $k, Z^{k}(G, A), B^{k}(G, A)$ and 
$C^{k}(G, A)$ will denote the groups of $k$-cocycles, $k$-coboundaries and $k$ cochains of $G$ over $A$ respectively. Also $\delta_{k}: C^{k}(G, A) \rightarrow C^{k+1}(G, A)$ will denote the $k$ th coboundary homomorphism. For definitions see [4]. For $\sigma \in Z^{k}(G, A),[\sigma]$ will denote the element of $H^{k}(G, A)$ to which $\sigma$ belongs. If $h \in H^{k}(G, A)$ and if $\sigma_{1}, \sigma_{2} \in Z^{n}(G, A)$ such that $\left[\sigma_{1}\right]=\left[\sigma_{2}\right]=h$ we say that $\sigma_{1}$ and $\sigma_{2}$ are cohomologous. Recall also that a sequence $\left\{h_{n}\right\}$ lies in $\lim _{n} \operatorname{inv}\left\{H^{k}\left(G, A_{n}\right)\right\}$ if $h_{n} \in H^{k}\left(G, A_{n}\right)$ for all $n$ and if $\pi_{n_{*}}\left(h_{n}\right)=h_{n-1}$ for all $n$. We shall use the same symbols $\pi_{n_{*}}$ and $\rho_{n_{*}}$ to denote the homomorphisms induced on the cohomology level and on the cochain level from $\pi_{n}$ and $\rho_{n}$. We now state the theorem.

TheOREM 2. For any integer $k \geqslant 0$ let $\left\{h_{n}\right\} \in \lim _{n} \operatorname{inv}\left\{H^{k}\left(G, A_{n}\right)\right\}$. Then for each $n$ there exists a $\sigma_{n} \in Z^{k}\left(G, A_{n}\right)$ such that

(1) $\pi_{n_{*}}\left(\sigma_{n}\right)=\sigma_{n-1}$,

(2) $\left[\sigma_{n}^{*}\right]=h_{n}$.

Proof. We use induction on $n$. Let $\sigma_{1}$ be any member of $h_{1}$. Now suppose we have succeeded in choosing a sequence $\sigma_{1}, \sigma_{2}, \ldots, \sigma_{n}$ satisfying conditions (1) and (2) for $1 \leqslant j \leqslant n$. Choose $\sigma_{n+1}^{\prime} \in h_{n+1}$. Since $\pi_{n+1 *}\left(\sigma_{n+1}^{\prime}\right)$ is cohomologous to $\sigma_{n}$ there exists a Borel function $\zeta_{n} \in C^{k-1}\left(G, A_{n}\right)$ such that $\pi_{n+1}\left(\sigma_{n+1}^{\prime}\right)+\delta_{k-1}(\zeta)=\sigma_{n}$. Let $\lambda_{n}: A_{n} \rightarrow A_{n+1}$ be a Borel cross section of $\pi_{n+1}$ such that $\lambda_{n}(1)=1$ (see [1]). Define $\psi_{n+1}$ to be the composition $\psi_{n+1}$ $=\lambda_{n} \zeta_{n}$. Note that $\psi_{n+1} \in C^{k-1}\left(G, A_{n+1}\right)$. Define $\sigma_{n+1}=\sigma_{n+1}^{\prime}+\delta_{k-1}\left(\psi_{n+1}\right)$. It immediately follows that $\left[\sigma_{n+1}\right]=h_{n+1}$ and

$$
\begin{aligned}
\pi_{n+1_{*}}\left(\sigma_{n+1}\right) & =\pi_{n+1_{*}}\left[\sigma_{n+1}^{\prime}+\delta_{k-1}\left(\psi_{n+1}\right)\right] \\
& =\pi_{n+1_{*}}\left(\sigma_{n+1}^{\prime}\right)+\delta_{k-1}\left(\zeta_{n}\right)=\sigma_{n} .
\end{aligned}
$$

Continuing in this manner we construct the desired sequence.

Corollary. The homomorphism $\Phi_{A}: H^{*}(G, A) \rightarrow \lim _{n} \operatorname{inv}\left\{H^{*}\left(G, A_{n}\right)\right\}$ is onto.

Proof. Let $\left\{h_{n}\right\} \in \lim _{n} \operatorname{inv}\left\{H^{k}\left(G, A_{n}\right)\right\}$ for some $k$. Let $\left\{\sigma_{n}\right\}$ be a sequence satisfying the conditions of Theorem 2. By condition (1) of Theorem 2 for any $\left(g_{1}, g_{2}, \ldots, g_{k}\right) \in G^{k}, \quad\left\{\sigma_{n}\left(g_{1}, g_{2}, \ldots, g_{k}\right)\right\} \in \lim _{n} \operatorname{inv}\left\{A_{n}\right\}$. Thus $\phi^{-1}\left(\left\{\sigma_{n}\left(g_{1}, \ldots, g_{k}\right)\right\}\right) \in A$ where $\phi$ is the topological isomorphism of Corollary 2 to Theorem 1. Now define $\sigma \in C^{k}(G, A)$ by $\sigma\left(g_{1}, \ldots, g_{k}\right)$ $=\phi^{-1}\left(\left\{\sigma_{n}\left(g_{1}, \ldots, g_{k}\right)\right\}\right)$ for $\left(g_{1}, \ldots, g_{k}\right) \in G^{k}$. It easily follows that $\sigma \in$ $Z^{k}(G, A)$ and if $h=[\sigma], \Phi_{A}(h)=\left\{h_{n}\right\}$. Thus $\Phi_{A}$ is onto.

We now turn to sufficient conditions for $\Phi_{A}$ to be one-to-one. First note that $B^{k}(G, A), Z^{k}(G, A)$ and $C^{k}(G, A)$ are all subsets of $A^{G^{k}}$, the set of all mappings of $G^{k}$ to $A$. As such they inherit topologies as subspaces of $A^{G^{k}}$ with the product topology.

THEOREM 3. If $B^{k}(G, A)$ is a closed subset of the topological space $C^{k}(G, A)$ for all $k$ then $\Phi_{A}$ is one-to-one and hence is an isomorphism.

Proof. Suppose the condition holds. Let $k \geqslant 0$. Let $h \in H^{k}(G, A)$ such that $\Phi_{A}(h)=0$. We show $h=0$.

By the exactness of the sequence 


$$
1 \rightarrow K_{n} \stackrel{i_{n}}{\rightarrow} A \stackrel{\rho_{n}}{\rightarrow} A_{n} \rightarrow 1
$$

and by [4] we obtain the exactness of

$$
\rightarrow H^{k}\left(G, K_{n}\right) \stackrel{i_{n}}{\rightarrow} H^{k}(G, A) \stackrel{\rho_{n}}{\rightarrow} H^{k}\left(G, A_{n}\right) \rightarrow .
$$

Since $\rho_{n_{*}}(h)=0$ for all $n$ there exists for each $n$ (by exactness) a $c_{n} \in$ $H^{k}\left(G, K_{n}\right)^{*}$ such that $i_{n_{*}}\left(c_{n}\right)=h$. Choose cocycles $\sigma \in h$ and $\beta_{n} \in c_{n}$ for each $n$. Since $i_{n_{*}}\left(\beta_{n}\right)$ is cohomologous to $h$ for each $n, i_{n}\left(\beta_{n}\right) \in \sigma+B^{k}(G, A)-\mathrm{a}$ closed subset of $C^{k}(G, A)$. For each $n, \beta_{n}$ maps $G^{k^{*}}$ into $K_{n}$. Since $K_{n} \subset U_{n}$ and $\left\{U_{n}\right\}$ is a decreasing base for the neighborhood system of the identity of $A$ we have

$$
\lim _{n \rightarrow \infty} i_{n_{*}} \beta_{n}\left(g_{1}, \ldots, g_{k}\right)=\lim _{n \rightarrow \infty} i_{n} \circ \beta_{n}\left(g_{1}, \ldots, g_{k}\right)=1
$$

for each $\left(g_{1}, g_{2}, \ldots, g_{k}\right) \in G^{k}$. Thus the sequence $\left\{i_{n} \beta_{n}\right\}$ converges to the trivial mapping, 0 , in the product topology. Thus by closure, $0 \in \sigma+$ $B^{k}(G, A)$. Thus $[\sigma]=0$. Thus $\Phi_{A}$ is one-to-one.

TheOREM 4. If $A$ is compact, $B^{k}(G, A)$ is closed in $C^{k}(G, A)$ and hence $\Phi_{A}$ is an isomorphism.

Proof. First define for each $k$ :

(1) $C_{N}^{k}(G, A)=\left\{f: G^{k} \rightarrow A \mid\right.$ if $g_{i}=1$ for some $\left.i, f\left(g_{1}, \ldots, g_{k}\right)=1\right\}$.

(2) $\delta_{N}^{k}: C_{N}^{k}(G, A) \rightarrow C_{N}^{k+1}(G, A)$ by

$$
\begin{aligned}
\delta_{k}^{N}\left(g_{1}, \ldots, g_{k+1}\right)= & g_{1} f\left(g_{2}, \ldots, g_{k+1}\right) \\
& +\sum_{i=1}^{k}(-1)^{i} f\left(g_{1}, \ldots, g_{i} \cdot g_{i+1}, \ldots, g_{k+1}\right) \\
& +(-1)^{k+1} f\left(g_{1}, \ldots, g_{k}\right) .
\end{aligned}
$$

(3) $Z_{N}^{k}(G, A)$-the kernel of $\delta_{k}^{N}$.

(4) $B_{N}^{k}(G, A)$-the image of $\delta_{k-1}^{N}$.

It is easily verified that $C_{N}^{k}(G, A)$ is closed in $A^{G^{k}}$ and hence is compact since $A$ is compact. It is also easily verified that $\delta_{k}^{N}$ is continuous for each $k$. Thus $B_{N}^{k}(G, A)$ and $Z_{N}^{k}(G, A)$ are both closed subsets of $C_{N}^{k}(G, A)$. Let $B$ denote the set of all Borel functions in $A^{G^{k}}$. Then

(5) $C^{k}(G, A)=C_{N}^{k}(G, A) \cap B$.

(6) $\delta_{k}=\delta_{k}^{N}$ restricted to $C^{k}(G, A)$.

(7) $Z^{k}(G, A)=Z_{N}^{k}(G, A) \cap B$.

(8) $B^{k}(G, A)=B_{N}^{k}(G, A) \cap B$.

Thus $B^{k}(G, A)$ is closed in $C^{k}(G, A)$. The result now follows from Theorem 3 .

THEOREM 5. If $A$ is connected $\Phi_{A}$ is an isomorphism.

Proof. Suppose $A$ is connected. By [5] $A$ splits as a direct sum of a compact group $C$ and a finite product $R^{p}$ of copies of the reals. Thus we have a splitting sequence $1 \rightarrow C \stackrel{i}{\rightarrow} A \stackrel{j}{\rightarrow} R^{p} \rightarrow 1$.

Note that $i(C)$ is invariant with respect to the action of $G$ since $R^{p}$ contains no nontrivial compact group. Thus $G$ induces an action on $C$ 
$\left(g \cdot c=i^{-1}(g \cdot i(c)), c \in C, g \in G\right)$ and an action on $R^{p}(g \cdot r=$ $j\left(g \cdot j^{-1}(r)\right), r \in R^{p}$ and $\left.g \in G\right)$. These actions are compatible with the homomorphisms $i$ and $j$.

Consider the collections $\left\{K_{n}\right\}$ and $\left\{U_{n}\right\}$ of Corollary 1 to Theorem 1. Note that $K_{n} \subset i(C)$ for each $n$ since $K_{n}$ is compact. For each $n$ define $H_{n}$ $=i^{-1}\left(K_{n}\right)$. Note that $C / H_{n}$ is a Lie group. This is so since $C / H_{n} \simeq$ $i(C) / K_{n}$-a closed subgroup of $A / K_{n}$.

For each $n$ let $V_{n}=i^{-1}\left(i(C) \cap U_{n}\right)$. Then $\left\{V_{n}\right\}$ and $\left\{H_{n}\right\}$ are decreasing sequences and C.

(1) $\left\{V_{n}\right\}$ is a decreasing base for the neighborhood system of the identity of

(2) $H_{n} \subset V_{n}$ for all $n$.

(3) $H_{n}$ is a $G$-invariant compact subgroup of $C$ for each $n$.

(4) $C / H_{n}$ is a Lie group for each $n$.

Thus Corollary 2 of Theorem 1 follows for Lie groups $\left\{C_{n}\right\}$ and sequences of homomorphisms $\left\{\rho_{n}\right\}$ and $\left\{\pi_{n}\right\}$ where $C_{n}=C / H_{n}, \rho_{n}: C \rightarrow C_{n}$ is the natural quotient map and $\pi_{n}\left(x \cdot C_{n}\right)=x \cdot C_{n-1}$ for $x \in C$, for all $n$. Furthermore we obtain for each $n$ the following commutative diagram:

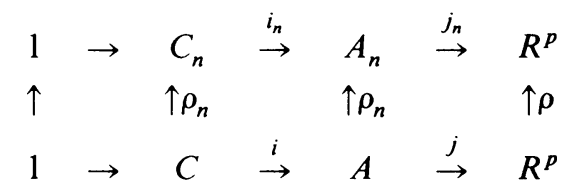

where $i_{n}\left(x \cdot H_{n}\right)=i(x) K_{n}$ for $x \cdot H_{n} \in C_{n}, j_{n}$ is the quotient map and $\rho$ is the identity.

By [4] we obtain the following commutative diagram of cohomology groups:

$$
\begin{array}{ccc}
\rightarrow H^{k}\left(G, C_{n}\right) & \stackrel{i_{n_{*}}}{\rightarrow} H^{k}\left(G, A_{n}\right) & \stackrel{j_{n_{*}}}{\rightarrow} H^{k}\left(G, R^{p}\right) \\
\uparrow \rho_{n_{*}} & \rho_{\rho_{n_{*}}} & \uparrow \rho_{*} \\
\rightarrow H^{k}(G, C) & \stackrel{i_{*}}{\rightarrow} H^{k}(G, A) & \stackrel{j_{*}}{\rightarrow} H^{k}\left(G, R^{p}\right) \\
\uparrow q_{n_{*}} & \uparrow q_{n_{*}} & \\
H^{k}\left(G, H_{n}\right) & \stackrel{i_{*}}{\rightarrow} H^{k}\left(G, K_{n}\right)
\end{array}
$$

where $q_{n}$ denote both the injections of $H_{n}$ into $C$ and of $K_{n}$ into $A$. Note that since $i: H_{n} \rightarrow K_{n}$ is an isomorphism so is $i_{*}: H^{k}\left(G, H_{n}\right) \rightarrow H^{k}\left(G . K_{n}\right)$.

We wish to show that $\Phi_{A}$ is one-to-one. Let $h \in H^{k}(G, A)$. Suppose $\Phi_{A}(h)=0$. Then $\rho_{n_{*}}(h)=0$ for all $n$. It suffices to show that $h=0$.

First note that $\rho_{1_{*}}(h)=0$. Hence $\rho_{*} j_{*}(h)=j_{1_{*}} \rho_{1_{*}}(h)=0$. Since $\rho_{*}$ is an isomorphism, $j_{*}(h) \stackrel{*}{=} 0$. Thus by exactness there exists a $c \in H^{k}(G, C)$ such that $i_{*}(c)=h$. Choose $\gamma \in Z^{n}(G, C)$ such that $\gamma \in c$. Let $\sigma=i_{*}(\gamma)$. Then $\sigma \in h$ and $\sigma \in i_{*}\left[Z^{k}(G, C)\right]$. For each $n$, since $\rho_{n_{*}}(h)=0$ we can choose $c_{n} \in H^{k}\left(G, K_{n}\right)$ such that $q_{n_{*}}\left(c_{n}\right)=h$. By commutativity we can choose $\beta_{n} \in Z^{k}\left(G, H_{n}\right)$ such that $i_{*} q_{n_{*}}\left(\beta_{n}\right) \in h$. Let $x_{n}=q_{n_{*}}\left(\beta_{n}\right)$. Then $i_{*}\left(\beta_{n}\right) \in$ 
$\sigma+B^{k}(G, A)$. Since both $\sigma$ and $i_{*}\left(x_{n}\right) \in i_{*}\left[Z^{k}(B, C)\right], i_{*}\left(x_{n}\right) \in \sigma+$ $i_{*}\left[B^{k}(B, C)\right]$.

Since $\bar{\beta}_{n}: G^{k} \rightarrow H_{n} \subset V_{n}$ and since $V_{n}$ is a decreasing base for the neighborhood system of the identity in $C$ we have that $\left\{\beta_{n}\right\} \rightarrow 0$ pointwise. Hence $\left\{x_{n}\right\}=\left\{q_{n}\left(\beta_{n}\right)\right\} \rightarrow 0$ pointwise. Hence $\left\{i_{*}\left(x_{n}\right)\right\} \rightarrow 0$ pointwise. Next observe that $i_{*}\left[B^{k}(G, C)\right]$ is closed in $C^{k}(G, A)$ in the product topology. This follows (using the same terminology as in the proof of Theorem 4) since $B_{N}^{k}(G, C)$ is a compact subset of $C_{N}^{k}(G, C)$ and $i_{*}$ is continuous. Thus $i_{*}\left[B_{N}^{k}(G, C)\right]$ is compact and hence closed in $C_{N}^{k}(G, A)$. Finally

$$
i_{*}\left[B^{k}(G, C)\right]=i_{*}\left[B_{N}^{k}(G, C)\right] \cap B
$$

is closed in $C^{k}(G, A)$. Thus $\sigma+i_{*}\left[B^{k}(G, C)\right]$ is closed and the sequence $\left\{i_{*}\left(x_{n}\right)\right\}$ in this set converges to 0 . Thus

$$
0 \in \sigma+i_{*}\left[B^{k}(G, C)\right] \subset \sigma+B^{k}(G, A) .
$$

Thus $h=0$. This establishes the theorem.

\section{REFERENCES}

1. L. Auslander and C. C. Moore, Unitary representations of solvable Lie groups, Mem. Amer. Math. Soc. no. 62(1966). MR34 \# 7723.

2. E. Hewitt and K. A. Ross, Abstract harmonic analysis. Vol. I: Structure of topological groups. Integration theory, group representations, Die Grundlehren der math. Wissenschaften, Band 115 , Academic Press, New York; Springer-Verlag, Berlin, 1963. MR28 \# 158.

3. D. Montgomery and L. Zippen, Topological transformation groups, Interscience, New York, 1955. MR17, 383.

4. C. C. Moore, Extensions and low dimensional cohomology theory of locally compact groups. I, Trans. Amer. Math. Soc. 113(1964), 40-63. MR30 \#2106.

5. W. Rudin, Fourier analysis on groups, Interscience Tracts in Pure and Appl. Math., no. 12, Interscience, New York, 1962. MR27 \#2808.

Department of Mathematics, Illinois State University, Normal, Illinois 61761 